\title{
Comparison of Clinical Trajectories before Initiation of Renal Replacement Therapy between Diabetic Nephropathy and Nephrosclerosis on the KDIGO Guidelines Heat Map
}

\author{
Masanori Abe, ${ }^{1}$ Kazuyoshi Okada, ${ }^{1}$ Noriaki Maruyama, ${ }^{1}$ Hiroyuki Takashima, \\ Osamu Oikawa, ${ }^{1}$ and Masayoshi Soma ${ }^{1,2}$ \\ ${ }^{1}$ Division of Nephrology, Hypertension and Endocrinology, Department of Internal Medicine, Nihon University School of Medicine, \\ Tokyo 173-8610, Japan \\ ${ }^{2}$ Division of General Medicine, Department of Internal Medicine, Nihon University School of Medicine, Tokyo 173-8610, Japan \\ Correspondence should be addressed to Masanori Abe; abe.masanori@nihon-u.ac.jp
}

Received 29 May 2015; Revised 23 August 2015; Accepted 5 October 2015

Academic Editor: Monika A. Niewczas

Copyright (C) 2016 Masanori Abe et al. This is an open access article distributed under the Creative Commons Attribution License, which permits unrestricted use, distribution, and reproduction in any medium, provided the original work is properly cited.

\begin{abstract}
This study investigated differences between the clinical trajectories of diabetic nephropathy and nephrosclerosis using the Kidney Disease: Improving Global Outcomes (KDIGO) heat map and the clinical characteristics between the two diseases at RRT initiation. This single-center, retrospective study enrolled 100 patients whose estimated glomerular filtration rate (eGFR) was $\geq 45 \mathrm{~mL} / \mathrm{min} / 1.73 \mathrm{~m}^{2}$ at their first visit and who were initiated on RRT. Fifty consecutive patients were assigned to each of the diabetic nephropathy and nephrosclerosis groups. All data for simultaneously measured eGFR and urinary albumin to creatinine ratio (UACR) were collected from first visit to RRT initiation and were plotted on the KDIGO heat map. Diabetic nephropathy was characterized by higher blood pressure and UACR and lower age, eGFR, and serum albumin levels compared with nephrosclerosis at RRT initiation. The vast majority of patients with diabetic nephropathy and eGFR $<60 \mathrm{~mL} / \mathrm{min} / 1.73 \mathrm{~m}{ }^{2}$ had concomitant macroalbuminuria, whereas for patients with nephrosclerosis, even when eGFR was $<45 \mathrm{~mL} / \mathrm{min} / 1.73 \mathrm{~m}{ }^{2}, \mathrm{many}$ still had normoalbuminuria or microalbuminuria. The rate of decline of eGFR was significantly faster in the diabetic nephropathy group than that in the nephrosclerosis group. The clinical trajectories of diabetic nephropathy and nephrosclerosis differed markedly on the KDIGO heat map.
\end{abstract}

\section{Background}

Chronic kidney disease (CKD) progressively increases the risk of end-stage kidney disease (ESKD) and cardiovascular disease in line with its severity [1]. The prevalence of ESKD is expected to rise steeply over the next few decades, driven by population ageing and the increasing prevalence of diabetes and hypertension [2-4]. Although renal replacement therapy (RRT), via dialysis or renal transplantation, is a potentially lifesaving treatment for patients with ESKD, it is costly.

Type 2 diabetes mellitus is among the leading causes of CKD, including ESKD, in both developed and developing countries; in various countries including the USA and Japan, type 2 diabetes mellitus accounts for nearly $50 \%$ of patients on incident dialysis [2,3]. In the USA in 2012, nephrosclerosis was the second most common primary disease after diabetic nephropathy [2], and in Japan in 2011, nephrosclerosis was the third most common primary disease (12.3\%) after diabetic nephropathy and chronic glomerulonephritis [5]. In relation to the aging of new dialysis patients, the percentage of patients who had nephrosclerosis and were newly started on dialysis continuously increased. Since about 2000 , the rate of increase in the annual number of new dialysis patients with chronic glomerulonephritis has been negative [5]. Therefore, in the future, nephrosclerosis will likely be the second most common primary disease in Japan as well as the USA. The management of diabetic nephropathy and nephrosclerosis is thus very important for helping prevent these patients from newly requiring RRT. 
The Kidney Disease: Improving Global Outcomes (KDIGO) Clinical Practice Guideline for the Evaluation and Management of CKD was released in January 2013 [6]. KDIGO recommends CKD classifications based on cause, glomerular filtration rate (GFR) category, and albuminuria category. The cause of $\mathrm{CKD}$ is considered because it provides important prognostic information and influences treatment decisions. Albuminuria and estimated GFR (eGFR) provide independent information regarding the risk of CKD progression, cardiovascular disease, and mortality. In addition, clinicians and researchers are advised to categorize patients using a heat map generated by composite rankings of relative risk. However, differences between the clinical trajectories for diabetic nephropathy and nephrosclerosis, the two major primary causes of $\mathrm{CKD}$, have not been revealed clearly on the KDIGO heat map.

Against this background, this retrospective study investigated differences between the clinical courses of diabetic nephropathy and nephrosclerosis using the KDIGO heat map and sought to determine how the clinical characteristics differed between the two diseases at the time of the RRT initiation.

\section{Methods}

This single-center, retrospective study, conducted between January 2011 and December 2013, was designed to compare the clinical courses of diabetic nephropathy and nephrosclerosis with respect to eGFR and the urinary albumin to creatinine ratio (UACR) in patients with CKD who were already receiving treatment from a nephrologist. Specifically, the study compared the clinical progression of the two diseases as represented by the heat map based on the prognosis of CKD by GFR and albuminuria category stated in the KDIGO 2012 Clinical Practice Guideline for the Evaluation and Management of Chronic Kidney Disease [6]. All data used in the analysis were collected from medical records. All study participants provided written informed consent, and the study protocol was approved by the Research Review Board of our University and conducted in accordance with the Declaration of Helsinki (Clinical Trial Registration Number: UMIN000017502).

Inclusion criteria were (1) patients who underwent RRT initiation at our hospital during January 2011 and December 2013 and (2) eGFR $\geq 45 \mathrm{~mL} / \mathrm{min} / 1.73 \mathrm{~m}^{2}$ at the first visit to our hospital. Exclusion criteria were $(1)$ age $<20$ years at RRT initiation, (2) RRT initiated due to acute kidney injury (AKI), and (3) primary cause of CKD other than diabetic nephropathy and nephrosclerosis (i.e., glomerulonephritis, cystic disease, or vasculitis). Diabetic nephropathy and nephrosclerosis were diagnosed by renal biopsy or medical history. Specifically, diabetic nephropathy was defined as diagnosis based on kidney biopsy $(n=15)$, on the presence of type 1 diabetes $(n=2)$, or on fulfillment of all the following criteria $(n=33)$ : (1) diabetes duration $\geq 10$ years; (2) clear presence of diabetic retinopathy; (3) no history of proteinuria or hematuria prior to the first visit; (4) other primary kidney disease, such as secondary, hereditary, cystic, or drug-induced kidney disease or vasculitis completely ruled out by blood work or imaging diagnostics. Nephrosclerosis was diagnosed by kidney biopsy $(n=10)$ or fulfillment of all of the following criteria $(n=40)$ : (1) no history of comorbid diabetes prior to the first visit or during the observational period; (2) duration of hypertension $\geq 10$ years; (3) no history of proteinuria or hematuria prior to the first visit; (4) presence of hypertensive retinopathy by fundus examination; and (5) other primary kidney disease, such as secondary, hereditary, cystic, drug-induced kidney disease, or vasculitis completely ruled out by blood work or imaging diagnostics. Subjects were assigned to either the diabetic nephropathy group or nephrosclerosis group at RRT initiation, with 50 consecutive subjects enrolled per group for a total of 100 subjects.

All data for simultaneously measured eGFR and UACR were used to monitor the clinical course from the first visit to RRT initiation and were collected from the medical records. These data were plotted on the heat map according to the KDIGO guidelines. Serum samples were assayed for creatinine $(\mathrm{sCr})$ at a central laboratory (Central Laboratory; SRL Co., Tokyo, Japan) with the enzymatic Cr assay method using a Japan electron Cr auto-analyzer (JCA-BM8060; JEOL Ltd., Tokyo, Japan) and enzyme solution (Preauto-S CRE-L; Sekisui Medical Co., Ltd., Tokyo, Japan). To assess urinary albumin excretion, we measured urinary concentrations of albumin and $\mathrm{Cr}$ (albumin/Cr ratio) in spot urine samples. Urinary albumin was measured using the immunoturbidimetric assay. Glomerular filtration rate was estimated using the modified, final recommended equation for Japanese patients issued by the Japanese Society of Nephrology-CKD Initiatives, as eGFR values obtained by this method are more accurate for Japanese patients with CKD [7]. The formula was as follows:

$$
\begin{aligned}
\operatorname{eGFR}\left(\mathrm{mL} / \mathrm{min} \text { per } 1.73 \mathrm{~m}^{2}\right) \\
=194 \times \mathrm{sCr}^{-1.094} \\
\quad \times \operatorname{age}^{-0.287}(\times 0.739 \text { for women }) .
\end{aligned}
$$

The composite ranking of relative risk by GFR and albuminuria levels was calculated according to the 2012 KDIGO guidelines using the following definitions: no CKD (green zone), G1A1 and G2A1; moderate risk (yellow zone), G1A2, G2A2, and G3aA1; high risk (orange zone), G1A3, G2A3, G3aA2, and G3bA1; and very high risk (red zone), G3aA3, G3bA2-3, all G4, and all G5 [6]. Blood pressure (BP) was measured at the outpatient clinic according to the Japanese Society of Hypertension 2009 guidelines [8]. Measurements were performed in duplicate every month using a sphygmomanometer (Nippon Colin, Tokyo, Japan) with the patient in a sitting position after a 5 min period of rest. Patients, particularly those with dietary restrictions, were given guidance on how to maintain their diet. Doses of antihypertensive agents, including angiotensin receptor blockers (ARBs), angiotensinconverting enzyme (ACE) inhibitors, calcium channel blockers, and diuretics, were adjusted during the study period to maintain the target BP level of $<130 / 80 \mathrm{mmHg}$.

2.1. Statistical Analysis. Data were analyzed on the basis of assigned groups and are expressed as the mean $\pm \mathrm{SD}$ 
TABLE 1: Clinical characteristics at the first visit in the two groups.

\begin{tabular}{|c|c|c|c|}
\hline & Diabetic nephropathy & Nephrosclerosis & $P$ value \\
\hline$n$ (male/female) & $50(34 / 16)$ & $50(35 / 15)$ & 0.833 \\
\hline Age (years) & $57.1 \pm 9.2$ & $65.6 \pm 8.4$ & $<0.0001$ \\
\hline Body mass index $\left(\mathrm{kg} / \mathrm{m}^{2}\right)$ & $24.7 \pm 2.5$ & $22.5 \pm 1.7$ & $<0.0001$ \\
\hline Systolic blood pressure ( $\mathrm{mmHg}$ ) & $149 \pm 8$ & $146 \pm 10$ & 0.038 \\
\hline Diastolic blood pressure ( $\mathrm{mmHg}$ ) & $86 \pm 7$ & $82 \pm 10$ & 0.012 \\
\hline Heart rate $(\mathrm{bpm})$ & $76 \pm 8$ & $76 \pm 7$ & 0.989 \\
\hline Serum creatinine $(\mathrm{mg} / \mathrm{dL})$ & $0.9 \pm 0.2$ & $1.1 \pm 0.2$ & $<0.0001$ \\
\hline eGFR $\left(\mathrm{mL} / \mathrm{min} / 1.73 \mathrm{~m}^{2}\right)$ & $65.6 \pm 10.5$ & $50.0 \pm 6.3$ & $<0.0001$ \\
\hline $\mathrm{UACR}(\mathrm{mg} / \mathrm{gCr})$ & $131[57,189]$ & $25[15,31]$ & $<0.0001$ \\
\hline Hemoglobin (g/dL) & $13.7 \pm 0.9$ & $13.5 \pm 0.7$ & 0.129 \\
\hline Serum albumin (g/dL) & $4.0 \pm 0.3$ & $4.0 \pm 0.2$ & 0.580 \\
\hline Type of diabetes (type 1/2) & $2 / 48$ & - & - \\
\hline Glycated hemoglobin (\%) & $7.6 \pm 0.6$ & $5.4 \pm 0.3$ & $<0.00001$ \\
\hline
\end{tabular}

Data are expressed as mean $\pm \mathrm{SD}$, median [interquartile range], or $n$.

GFR, glomerular filtration rate; UACR, urinary albumin to creatinine ratio.

or median [interquartile range], as appropriate. Continuous variables were compared using Student's $t$-test or the MannWhitney $U$ test, and categorical variables were compared by the chi-square or Fisher's exact test as appropriate to the data distribution. To analyze the time course changes in eGFR, we fitted scatterplot smoothing curves to all the eGFR measures for all the patients in each group. Then, we used the Mann-Whitney $U$ test to compare the eGFR decline $\left(\mathrm{mL} / \mathrm{min} / 1.73 \mathrm{~m}^{2}\right.$ per year) between the groups. The eGFR time course data within groups were analyzed by repeatedmeasures analysis of variance (ANOVA), while changes between the two groups were analyzed by two-way ANOVA followed by Dunnett's test. To analyze the time course changes in albuminuria, we fitted scatterplot smoothing curves to all UACR measures for all the patients in each group. Then, we used the Mann-Whitney $U$ test to compare the regression coefficients between the groups. Statistical significance was set at $P<0.05$. All analyses were performed using JMP ver. 11 software (SAS Institute Ltd., Cary, NC, USA).

\section{Results}

3.1. Study Population and Characteristics at the First Visit and RRT Initiation. The clinical characteristics of the patients at the first visit are shown in Table 1. At the first visit, mean age was significantly higher in the nephrosclerosis group than in the diabetic nephropathy group. Body mass index (BMI) was significantly higher in the diabetic nephropathy group. Although there was no significant difference in heart rate, both systolic and diastolic BP was significantly higher in the diabetic nephropathy group. Serum Cr level was significantly lower and eGFR was significantly higher in the diabetic nephropathy group. There was no significant difference in hemoglobin or serum albumin level between the groups. Mean glycated hemoglobin level was $7.6 \pm 0.6 \%$ in the diabetic nephropathy group.

The patients' clinical characteristics and medications being taken at RRT initiation are shown for each group in
Table 2. Mean retrospective observational period and mean time of simultaneous measurement of eGFR and albuminuria did not significantly differ between the groups. At RRT initiation, mean age was significantly higher in the nephrosclerosis group than in the diabetic nephropathy group. BMI was significantly higher in the diabetic nephropathy group. Although there was no significant difference in heart rate, both systolic and diastolic BP was significantly higher in the diabetic nephropathy group. There was a significantly higher occurrence of cardiovascular comorbidity, in particular ischemic heart disease, in the diabetic nephropathy group. All patients had hypertension and were taking antihypertensive medication, with renin-angiotensin system (RAS) inhibitors including ARBs, ACE inhibitors, and direct renin inhibitors being the most common, followed by calcium channel blockers. Although 49 patients in the diabetic nephropathy group and 46 patients in the nephrosclerosis group had used diuretics, thiazide diuretics were used by only 3 patients in the diabetic nephropathy group and none in the nephrosclerosis group; other patients used loop diuretics. Although there was no significant difference in the type of antihypertensive agents used in the two groups, the number of such agents used per person was significantly greater in the diabetic nephropathy group.

3.2. Laboratory Data at RRT Initiation. The final data set collected before RRT initiation is shown in Table 3. The nephrosclerosis group had significantly higher serum $\mathrm{Cr}$ levels and lower eGFR values than the diabetic nephropathy group. The diabetic nephropathy group had a significantly higher UACR and a significantly lower serum albumin level. The diabetic nephropathy group had significantly higher triglyceride, N-terminal pro-brain natriuretic peptide (NTproBNP), and C-reactive protein (CRP) levels and significantly lower high-density lipoprotein (HDL)-cholesterol levels. Hemoglobin level did not differ significantly between the groups. The glycated hemoglobin level was significantly 
TABLE 2: Clinical characteristics and medications at the initiation of RRT in the two groups.

\begin{tabular}{|c|c|c|c|}
\hline & Diabetic nephropathy & Nephrosclerosis & $P$ value \\
\hline$n$ (male/female) & $50(34 / 16)$ & $50(35 / 15)$ & 0.833 \\
\hline Age (years) & $67.2 \pm 9.6$ & $78.8 \pm 6.4$ & $<0.0001$ \\
\hline Observational periods (months) & $115 \pm 57$ & $122 \pm 35$ & 0.447 \\
\hline Measurement times (/year) & $4.4 \pm 2.5$ & $4.4 \pm 2.6$ & 0.955 \\
\hline Measurement times (/person) & $34 \pm 11$ & $37 \pm 10$ & 0.109 \\
\hline Body mass index $\left(\mathrm{kg} / \mathrm{m}^{2}\right)$ & $24.8 \pm 2.5$ & $22.1 \pm 1.7$ & $<0.0001$ \\
\hline Systolic blood pressure (mmHg) & $147 \pm 15$ & $137 \pm 9$ & $<0.0001$ \\
\hline Diastolic blood pressure ( $\mathrm{mmHg}$ ) & $80 \pm 12$ & $73 \pm 10$ & 0.0003 \\
\hline Heart rate $(\mathrm{bpm})$ & $77 \pm 7$ & $76 \pm 8$ & 0.541 \\
\hline \multicolumn{4}{|l|}{ Mode of renal replacement therapy $\%(n)$} \\
\hline Hemodialysis & $92(46)$ & $92(46)$ & - \\
\hline Peritoneal dialysis & $8(4)$ & $8(4)$ & - \\
\hline Kidney transplantation & $0(0)$ & $0(0)$ & - \\
\hline Cardiovascular comorbidities \% $(n)$ & $34(17)$ & $18(9)$ & 0.069 \\
\hline Ischemic heart disease & $28(14)$ & $12(6)$ & 0.046 \\
\hline Cerebrovascular disease & $6(3)$ & $4(2)$ & 0.650 \\
\hline Peripheral artery disease & $4(2)$ & $2(1)$ & 0.562 \\
\hline Diabetic retinopathy \% $(n)$ & $100(50)$ & - & - \\
\hline \multicolumn{4}{|l|}{ Medication \% (n) } \\
\hline \multicolumn{4}{|l|}{ Antihypertensive agents } \\
\hline Angiotensin receptor blockers & $98(49)$ & $90(45)$ & 0.093 \\
\hline Angiotensin-converting enzyme inhibitors & $14(7)$ & $4(2)$ & 0.082 \\
\hline Direct renin inhibitors & $8(4)$ & $2(1)$ & 0.173 \\
\hline Calcium channel blockers & $98(49)$ & $94(47)$ & 0.312 \\
\hline Diuretics & $98(49)$ & $92(46)$ & 0.172 \\
\hline$\beta$-blockers & $30(15)$ & $14(7)$ & 0.054 \\
\hline$\alpha$-blockers & $30(15)$ & $14(7)$ & 0.054 \\
\hline Number of antihypertensive agents (per person) & $3.76 \pm 0.2$ & $3.10 \pm 0.1$ & 0.0006 \\
\hline \multicolumn{4}{|l|}{ Antidiabetic agents } \\
\hline Insulin & $38(19)$ & - & - \\
\hline Oral hypoglycemic agents & $58(29)$ & - & - \\
\hline Diet therapy alone & $4(2)$ & - & - \\
\hline Erythropoiesis stimulating agents & $100(50)$ & $98(49)$ & 0.319 \\
\hline Statins & $80(40)$ & $78(39)$ & 0.808 \\
\hline Active vitamin $\mathrm{D}$ & $94(47)$ & $92(46)$ & 0.698 \\
\hline
\end{tabular}

Data are expressed as mean $\pm \mathrm{SD}, \%$, or $n$.

decreased at RRT initiation compared with that at the first visit in the diabetic nephropathy group $(P<0.0001)$.

3.3. Time Course of eGFR Decline and Urinary Albumin Excretion Rate. Figure 1 shows the eGFR trajectories of individuals in the diabetic nephropathy (a) and nephrosclerosis (b) groups. The mean eGFR slopes from first visit to RRT initiation for the diabetic nephropathy group and nephrosclerosis group were $-6.6 \pm 2.4$ and $-3.6 \pm 1.2 \mathrm{~mL} / \mathrm{min} / 1.73 \mathrm{~m}^{2}$ per year, respectively $(P<0.0001)$. The duration between the observation of eGFR $<45 \mathrm{~mL} / \mathrm{min} / 1.73 \mathrm{~m}^{2}$ and RRT initiation was $59 \pm 26$ months in the diabetic nephropathy group and $94 \pm 28$ months in the nephrosclerosis group, showing a significant difference between the groups $(P<0.0001)$.
Furthermore, the rates of decline in eGFR in the diabetic nephropathy group and nephrosclerosis group were $-9.9 \pm$ 5.3 and $-4.8 \pm 2.2 \mathrm{~mL} / \mathrm{min} / 1.73 \mathrm{~m}^{2}$ per year, respectively $(P<$ $0.0001)$. Figure 2 shows the UACR trajectories of individuals in the diabetic nephropathy (a) and nephrosclerosis (b) groups. The regression coefficient was $-23.3[-34$ to -13$]$ in the diabetic nephropathy group and -4.7 [ -9.7 to -2.2$]$ in the nephrosclerosis group, again showing a significant difference between the groups $(P<0.0001)$.

3.4. Clinical Course on the KDIGO Heat Map. Figure 3 shows all plotted data for simultaneously measured eGFR and UACR from the first visit to our hospital to final data collection before RRT initiation in the two groups. In 
TABLE 3: Laboratory data before the initiation of RRT in the two groups.

\begin{tabular}{lccc}
\hline Variables & Diabetic nephropathy & Nephrosclerosis & $P$ value \\
\hline Serum creatinine $(\mathrm{mg} / \mathrm{dL})$ & $8.8 \pm 1.4$ & $9.9 \pm 1.6$ & 0.004 \\
eGFR $\left(\mathrm{mL} / \mathrm{min} / 1.73 \mathrm{~m}^{2}\right)$ & $5.5 \pm 1.1$ & $4.2 \pm 0.8$ & $<0.0001$ \\
UACR $(\mathrm{mg} / \mathrm{gCr})$ & $3000[2084,4184]$ & $972[490,1830]$ & $<0.0001$ \\
Hemoglobin $(\mathrm{g} / \mathrm{dL})$ & $10.1 \pm 0.9$ & $10.7 \pm 0.9$ & 0.290 \\
Serum albumin $(\mathrm{g} / \mathrm{dL})$ & $3.3 \pm 0.6$ & $3.7 \pm 0.4$ & 0.0012 \\
Total cholesterol $(\mathrm{mg} / \mathrm{dL})$ & $173 \pm 38$ & $167 \pm 35$ & 0.341 \\
HDL-cholesterol $(\mathrm{mg} / \mathrm{dL})$ & $45 \pm 13$ & $52 \pm 13$ & 0.013 \\
Triglyceride $(\mathrm{mg} / \mathrm{dL})$ & $141[96,178]$ & $94[76,127]$ & $<0.0001$ \\
Glycated hemoglobin $(\%)$ & $6.5 \pm 0.7$ & $5.6 \pm 0.4$ & $<0.0001$ \\
NT-proBNP $(\mathrm{pg} / \mathrm{mL})$ & $2670[1531,7209]$ & $1298[594,3226]$ & 0.021 \\
C-reactive protein $(\mathrm{mg} / \mathrm{dL})$ & $0.17[0.10,0.29]$ & $0.09[0.03,0.14]$ & $<0.0001$ \\
\hline
\end{tabular}

Data are expressed as mean \pm SD or median [interquartile range]. eGFR, estimated glomerular filtration rate; HDL, high-density lipoprotein; NT-proBNP, Nterminal pro-brain natriuretic peptide; UACR, urinary albumin to creatinine ratio.

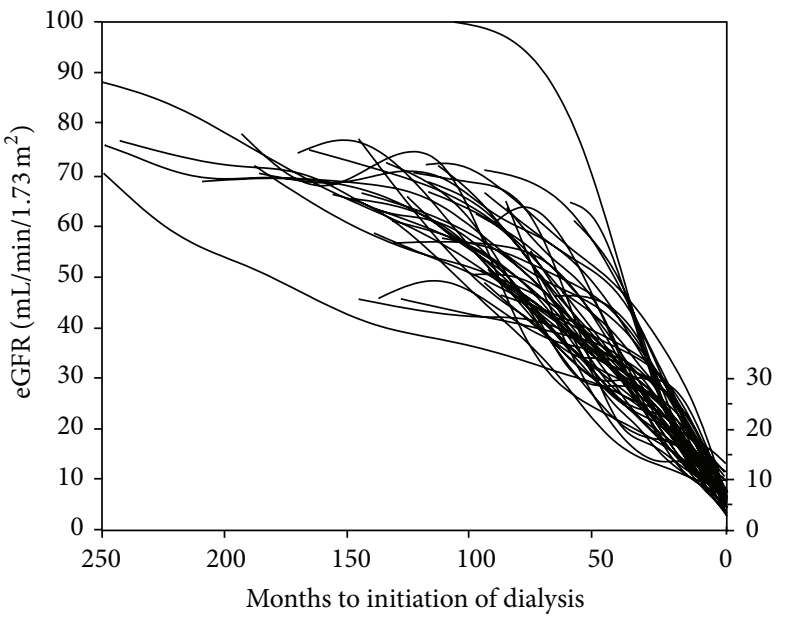

(a)

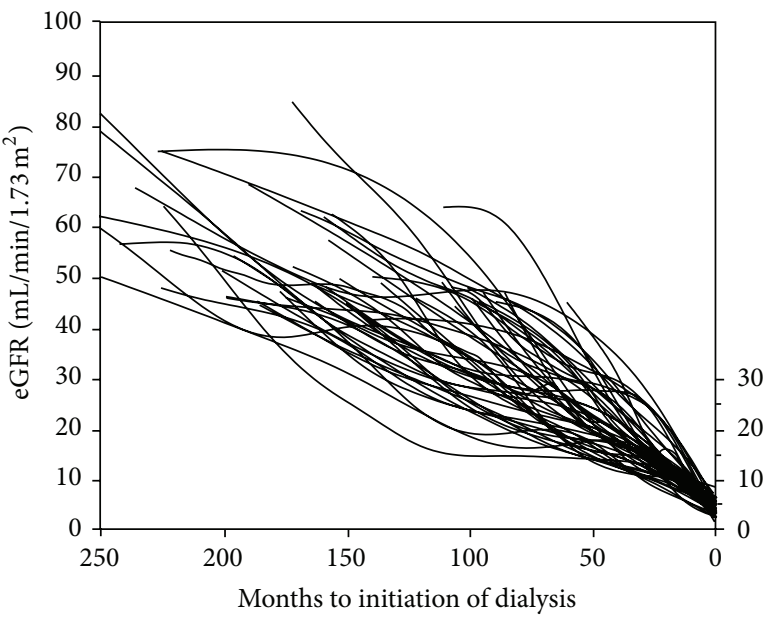

(b)

FIGURE 1: Estimated glomerular filtration rate (eGFR) trajectories with individual slopes during the observation period in the two groups. (a) Smoothing curve for the diabetic nephropathy group; (b) smoothing curve for the nephrosclerosis group.

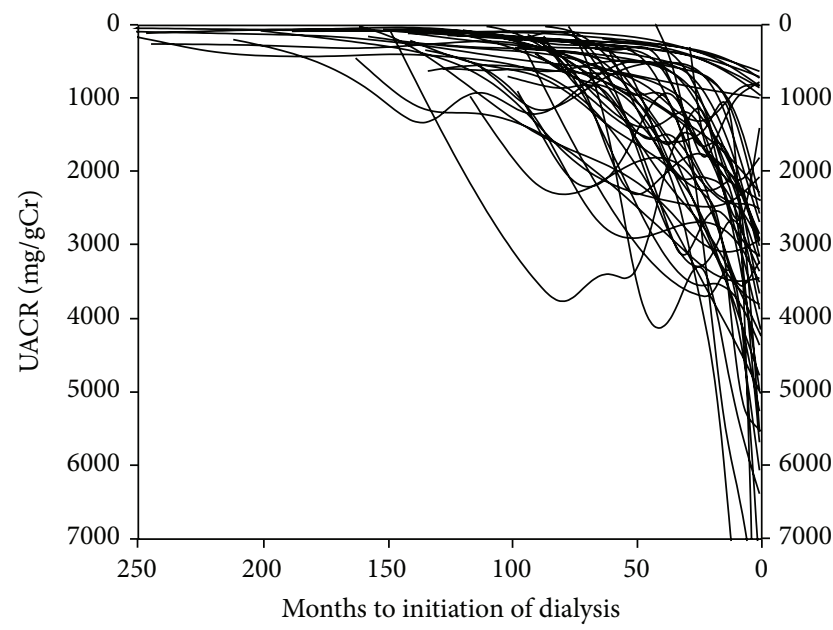

(a)

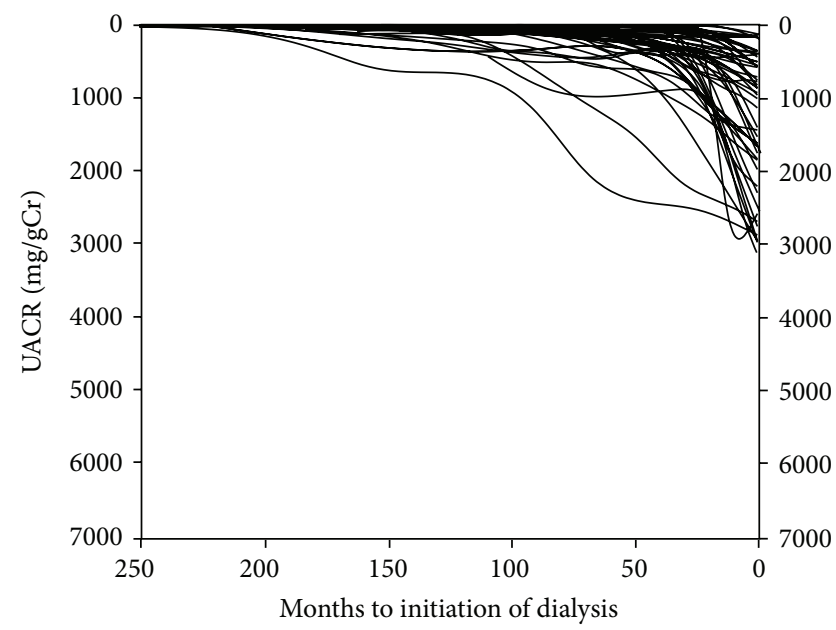

(b)

FIGURE 2: Urinary albumin to creatinine ratio (UACR) trajectories with individual slopes during the observation period in the two groups. (a) Smoothing curve for the diabetic nephropathy group; (b) smoothing curve for the nephrosclerosis group. 


\begin{tabular}{|c|c|c|}
\hline \multicolumn{3}{|c|}{ Albuminuria stage } \\
\hline $\begin{array}{c}\text { Optimal and } \\
\text { high-normal }\end{array}$ & A2 & A3 \\
\hline$<29$ & $30-299$ & $\begin{array}{c}\text { Very high } \\
\text { and nephrotic }\end{array}$ \\
\hline
\end{tabular}

$\operatorname{UACR}(\mathrm{mg} / \mathrm{g})$

\begin{tabular}{|c|c|c|c|}
\hline & G1 & $\begin{array}{c}\text { High and } \\
\text { optimal }\end{array}$ & $90-120$ \\
\hline & G2 & Mild & $60-90$ \\
\cline { 2 - 4 } & G3a & $\begin{array}{c}\text { Mild- } \\
\text { moderate }\end{array}$ & $45-59$ \\
$\begin{array}{c}\text { GFR } \\
\text { stage }\end{array}$ & G3b & $\begin{array}{c}\text { Moderate- } \\
\text { severe }\end{array}$ & $30-44$ \\
\hline & G4 & Severe & $15-29$ \\
\hline & G5 & $\begin{array}{c}\text { Kidney } \\
\text { failure }\end{array}$ & $<15$ \\
\hline
\end{tabular}

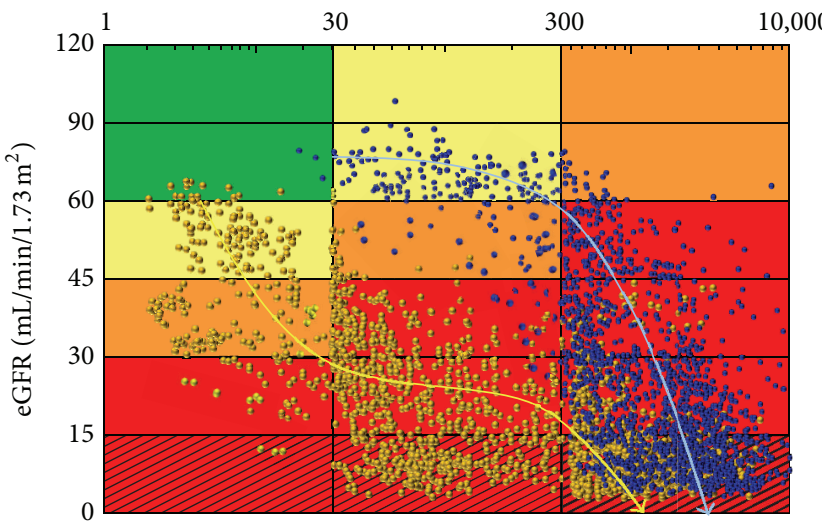

FIGURE 3: Data of simultaneously measured eGFR and UACR from the first visit to RRT initiation in the diabetic nephropathy group and nephrosclerosis group as represented on the KDIGO heat map. Blue and yellow bold arrows indicate clinical trajectories of the diabetic nephropathy and nephrosclerosis groups, respectively. eGFR, estimated glomerular filtration rate; GFR, glomerular filtration rate; KDIGO, Kidney Disease: Improving Global Outcomes; UACR, urinary albumin to creatinine ratio.

the diabetic nephropathy group, only 3 patients $(6 \%)$ were plotted into the no CKD (G2A1) category at the first visit. Five patients $(10 \%)$ and 2 patients $(4 \%)$ were plotted into the high risk G3aA2 and G2A3 categories, respectively; the remaining 40 patients $(80 \%)$ were plotted into the moderate risk category. In the nephrosclerosis group, 3 patients (6\%) were plotted into the no CKD (G2A1) category at the first visit. However, 6 patients (12\%) were plotted into the high risk category (G3aA2), and the remaining 41 patients $(82 \%)$ were plotted into the moderate risk category (G3aAl). None of the patients in the nephrosclerosis group were plotted into G2A2 or G2A3, unlike many patients in the diabetic nephropathy group. In the diabetic nephropathy group, risk categories progressed from moderate or high risk to very high risk when GFR was reduced to $<60 \mathrm{~mL} / \mathrm{min} / 1.73 \mathrm{~m}^{2}$. In other words, when eGFR was reduced to $<60 \mathrm{~mL} / \mathrm{min} / 1.73 \mathrm{~m}^{2}$, albuminuria showed progression from the A2 to A3 stage. Moreover, the eGFR decline resulted in further elevation of albuminuria up to 3000 (interquartile range, 2084 to 4184$) \mathrm{mg} / \mathrm{gCr}$ at RRT initiation. All cases underwent RRT initiation at the G5A3 stage. On the other hand, in the nephrosclerosis group, when eGFR was reduced to $<45 \mathrm{~mL} / \mathrm{min} / 1.73 \mathrm{~m}^{2}$ and albuminuria had progressed from the A1 to A2 stage, the risk category changed from moderate or high risk to very high risk. Thereafter, eGFR gradually decreased and albuminuria gradually increased. Eight patients (16\%) were started on RRT while remaining at the $\mathrm{A} 2$ stage, whereas the other 42 patients (84\%) had progressed to A3 at RRT initiation.

\section{Discussion}

These results reveal that the clinical courses of the two primary causative diseases of $\mathrm{CKD}$-diabetic nephropathy and nephrosclerosis-differ considerably when represented on the KDIGO heat map. As shown in Figure 3, diabetic nephropathy and nephrosclerosis showed contrasting characteristic courses. Furthermore, there was a significant difference in the rate of decline of eGFR between patients with diabetic nephropathy and those with nephrosclerosis. This indicates that even when eGFR levels are comparable, the subsequent progression in diabetic nephropathy would be more rapid compared with that for nephrosclerosis. It is recommended that patients be referred to nephrology at stage 4 CKD to prepare for RRT. Nephrologists and general physicians should be aware of the different clinical courses of these two CKDs and should aim to differentiate the causes of CKD upon physician examination.

Declines in eGFR, such as a $30 \%$ reduction over 2 years, were reported to be strongly and consistently associated with the risks for ESKD and mortality and have been considered an alternative endpoint for CKD progression [9]. Although the traditional view of kidney function decline in CKD is a steady linear decline (or slope), albeit at different rates among individuals, recent studies have evaluated the trajectories of decline and have shown they are often not linear [10-12]. The average overall rate of decline reported in 1441 adult individuals with stage 3-5 CKD was $1.47 \mathrm{~mL} / \mathrm{min} / 1.73 \mathrm{~m}^{2}$; however, the rate was faster in individuals with eGFR < $30 \mathrm{~mL} / \mathrm{min} / 1.73 \mathrm{~m}^{2}$ and accelerated in the year before the development of ESKD [10]. Individuals with steeper trajectories were more likely to have been hospitalized and to receive a diagnosis of AKI during hospitalization [12]. Although the patients who began RRT due to AKI were not included in the present study, diabetic nephropathy might be predisposed to rapid decline compared with nephrosclerosis. These findings highlight the heterogeneity of the rates of decline of eGFR and 
should lead to more individualized approaches to preparation for ESKD and transplant referral. Further studies should focus on identifying risk factors for the rapid decline of eGFR to allow for more timely intervention, as trajectories according to the primary disease of CKD have not been considered in previous studies.

Recently, the prognostic significance of identifying individuals with diabetes and an early decline (starting at $\left.<60 \mathrm{~mL} / \mathrm{min} / 1.73 \mathrm{~m}^{2}\right)$ in GFR $\left(>3.5 \mathrm{~mL} / \mathrm{min} / 1.73 \mathrm{~m}^{2}\right.$ per year) that is over and above what would be expected with aging alone has been highlighted, with this early decline being linked to the development of ESKD in type 1 diabetes $[13,14]$. Krolewski reported that $25 \%$ of patients with diabetes can be considered to have rapid progressive renal decline (eGFR slope $<-7 \mathrm{~mL} / \mathrm{min} /$ year) and these patients progressed to ESKD within 2-10 years. Another $25 \%$ showed moderate progressive renal decline (eGFR slope -7 to $-3 \mathrm{~mL} / \mathrm{min} /$ year), and most of them progressed or will progress to ESKD within $10-30$ years. The remaining patients $(50 \%)$ will have slow or no progressive renal decline and few may progress to ESKD during 30 years of follow-up [15]. Furthermore, the prevalence rate of patients showing such renal decline is $10 \%, 32 \%$, and $50 \%$ among patients with normoalbuminuria, microalbuminuria, and proteinuria, respectively [16]. In the present study, the GFR decline rate in the diabetic nephropathy group was $-6.6 \mathrm{~mL} / \mathrm{min} / 1.73 \mathrm{~m}^{2}$ per year during 115 months overall. However, when the eGFR declined to less than $45 \mathrm{~mL} / \mathrm{min} / 1.73 \mathrm{~m}^{2}$, the decline rate accelerated to $-9.9 \mathrm{~mL} / \mathrm{min} / 1.73 \mathrm{~m}^{2}$ per year.

However, some studies have shown microalbuminuria remission rates of $21-64 \%$ in patients with diabetes [17-22]. These high rates of microalbuminuria remission have been linked to the use of RAS inhibitors in some studies. Currently, there is known to be a four- to fivefold magnitude increase in the risk for ESKD in patients with type 1 diabetes or type 2 diabetes and microalbuminuria [23]. However, many of our patients with ESKD due to diabetic nephropathy had resistant hypertension and higher blood pressures compared with those with ESKD due to nephrosclerosis, despite taking significantly larger numbers of antihypertensive agents. In the absence of antihypertensive therapy, GFR may decrease by $10-15 \mathrm{~mL} / \mathrm{min}$ per year during stage 4 , which is characterized by clinically detectable proteinuria, hypertension, and declining GFR [24]. Therefore, our data demonstrate that, in patients with diabetic nephropathy, the inhibition of progression from microalbuminuria to macroalbuminuria is important for preventing progression to ESKD. Although RAS inhibitors remain the cornerstone of therapy, the management of patients who do not respond to them remains an issue.

Recent studies demonstrated that normoalbuminuric renal insufficiency is not uncommon for diabetic patients, especially those with type 2 diabetes [25]. There are several possible pathogenic mechanisms that may account for the development of normoalbuminuric renal insufficiency. Renal ischemia due to intrarenal arteriosclerosis and disproportionately advanced tubulointerstitial lesions, despite minor diabetic glomerular lesions, which denote the presence of diabetic kidney lesions as well as nephrosclerosis, are likely to be related to the development of normoalbuminuric renal insufficiency $[26,27]$. The clinical characteristics of such patients include older age, female predilection, shorter duration of diabetes, lower prevalence of hypertension, smoking, previous cardiovascular disease, and antihypertensive agents including RAS inhibitors, lower levels of glycated hemoglobin, and higher levels of HDL-cholesterol [28-30]. The diabetic nephropathy group in the present study included patients in whom diabetic nephropathy showed a typical clinical course, since none had normoalbuminuric renal insufficiency.

We recognize that our study is limited by the diagnostic methods used for diabetic nephropathy and nephrosclerosis. Moreover, there were only 17 and 10 biopsy-proven patients in the diabetic nephropathy and nephrosclerosis groups, respectively. Therefore, patients with chronic glomerulonephritis might have been included in the diabetic nephropathy group. However, all patients in the diabetic nephropathy group had diabetic retinopathy and a prolonged duration of diabetes. Therefore, we believe that the diagnosis of diabetic nephropathy was fairly certain in our patients. A second limitation is that the frequency of simultaneous measurement of eGFR and UACR was lower for the duration from the first visit to an eGFR of $45 \mathrm{~mL} / \mathrm{min} / 1.73 \mathrm{~m}^{2}$, because in Japan, many patients are commonly followed by a general physician. Patients were thereafter treated by a nephrologist only when eGFR decreased to $<45-30 \mathrm{~mL} / \mathrm{min} / 1.73 \mathrm{~m}^{2}$. Therefore, there was less data for the duration in the moderate and high risk categories than for the very high risk category, and the precise duration from the no CKD to moderate risk categories (G2A2 and G3aA1) could not be determined. Although some patients with diabetic nephropathy rapidly progress to ESKD, these patients were excluded from the present analysis because they had less data available for simultaneous measurements and most of them already had eGFR $<30 \mathrm{~mL} / \mathrm{min} / 1.73 \mathrm{~m}^{2}$ at the first visit. Moreover, we could not clarify the eGFR and UACR trajectories of patients who had no or minimal decline in eGFR over the study period, since our study design allowed for only the investigation of subjects that ultimately progressed to ESKD. Lastly, the sample size was relatively small, and our study was retrospective. However, if this study were to be performed as a prospective study, we would need a relatively long period to complete it, as the endpoint of the study is RRT initiation. Nevertheless, additional studies are necessary to more firmly establish whether the risk categories of the KDIGO classification precisely reflect prognosis; not only the requirements for RRT but also cardiovascular events should be considered as endpoints since the risk categories of the KDIGO classification have three distinct indications, namely, risks for ESKD, cardiovascular events, and all-cause mortality.

\section{Conclusions}

This retrospective analysis showed that the clinical trajectories to RRT initiation on the KDIGO heat map differed between diabetic nephropathy and nephrosclerosis. The rate 
of decline of eGFR in the diabetic nephropathy group was significantly faster than that in the nephrosclerosis group. Therefore, identification of the primary disease of CKD by kidney biopsy might be important for determining the likelihood of progression to ESKD. Furthermore, compared with nephrosclerosis, diabetic nephropathy was characterized at RRT initiation by higher BMI, higher systolic and diastolic BPs, and higher CRP, NT-proBNP, and albuminuria levels as well as lower age and serum albumin levels. Further studies are needed to clarify the factors that influence the progression to ESKD.

\section{Conflict of Interests}

The authors declare no conflict of interests.

\section{Authors' Contribution}

Masanori Abe conceived of the study and participated in its design, advised throughout the study and at final approval, and helped draft the paper. Kazuyoshi Okada, Noriaki Maruyama, Osamu Oikawa, and Hiroyuki Takashima participated in its design and coordination, drafted the paper, and performed statistical analysis. Masayoshi Soma reviewed the study design and revised the paper. All authors read and approved the final paper.

\section{References}

[1] A. S. Levey, P. E. de Jong, J. Coresh et al., "The definition, classification, and prognosis of chronic kidney disease: a KDIGO Controversies Conference report," Kidney International, vol. 80, no. 1, pp. 17-28, 2011.

[2] A. J. Collins, R. N. Foley, C. Herzog et al., "US renal data system 2012 annual data report," American Journal of Kidney Diseases, vol. 61, no. 1, supplement 1, article A7, pp. e1-e476, 2013.

[3] M. Abe and K. Kalantar-Zadeh, "Haemodialysis-induced hypoglycaemia and glycaemic disarrays," Nature Reviews Nephrology, vol. 11, no. 5, pp. 302-313, 2015.

[4] S. L. White, S. J. Chadban, S. Jan, J. R. Chapman, and A. Cass, "How can we achieve global equity in provision of renal replacement therapy?" Bulletin of the World Health Organization, vol. 86, no. 3, pp. 229-237, 2008.

[5] S. Nakai, Y. Watanabe, I. Masakane et al., "Overview of regular dialysis treatment in Japan (as of 31 December 2011)," Therapeutic Apheresis and Dialysis, vol. 17, no. 6, pp. 567-611, 2013.

[6] Kidney Disease Improving Global Outcomes (KDIGO) CKD Work Group, "KDIGO 2012 clinical practice guideline for the evaluation and management of chronic kidney disease," Kidney International Supplements, vol. 3, no. 5, pp. 1-150, 2013.

[7] S. Matsuo, E. Imai, M. Horio et al., "Revised equations for estimated GFR from serum creatinine in Japan," American Journal of Kidney Diseases, vol. 53, no. 6, pp. 982-992, 2009.

[8] Japanese Society of Hypertension, "Japanese Society of Hypertension guidelines for the management of hypertension (JSH 2009)," Hypertension Research, vol. 32, no. 4, pp. 4-107, 2009.

[9] J. Coresh, T. C. Turin, K. Matsushita et al., "Decline in estimated glomerular filtration rate and subsequent risk of end-stage renal disease and mortality," The Journal of the American Medical Association, vol. 311, no. 24, pp. 2518-2531, 2014.
[10] J. G. Heaf and L. S. Mortensen, "Uraemia progression in chronic kidney disease stages 3-5 is not constant," Nephron: Clinical Practice, vol. 118, no. 4, pp. c367-c374, 2011.

[11] L. Li, B. C. Astor, J. Lewis et al., "Longitudinal progression trajectory of GFR among patients with CKD," American Journal of Kidney Diseases, vol. 59, no. 4, pp. 504-512, 2012.

[12] A. M. O’Hare, A. Batten, N. R. Burrows et al., "Trajectories of kidney function decline in the 2 years before initiation of longterm dialysis," American Journal of Kidney Diseases, vol. 59, no. 4, pp. 513-522, 2012.

[13] R. J. Macisaac, E. I. Ekinci, and G. Jerums, "Markers of and risk factors for the development and progression of diabetic kidney disease," American Journal of Kidney Diseases, vol. 63, no. 2, pp. S39-S62, 2014.

[14] J. Skupien, J. H. Warram, A. M. Smiles et al., "The early decline in renal function in patients with type 1 diabetes and proteinuria predicts the risk of end-stage renal disease," Kidney International, vol. 82, no. 5, pp. 589-597, 2012.

[15] A. S. Krolewski, "Progressive renal decline: the new paradigm of diabetic nephropathy in type 1 diabetes," Diabetes Care, vol. 38, no. 6, pp. 954-962, 2015.

[16] A. S. Krolewski, M. A. Niewczas, J. Skupien et al., "Early progressive renal decline precedes the onset of microalbuminuria and its progression to macroalbuminuria," Diabetes Care, vol. 37, no. 1, pp. 226-234, 2014.

[17] B. A. Perkins, L. H. Ficociello, K. H. Silva, D. M. Finkelstein, J. H. Warram, and A. S. Krolewski, "Regression of microalbuminuria in type 1 diabetes," The New England Journal of Medicine, vol. 348, no. 23, pp. 2285-2293, 2003.

[18] P. Hovind, L. Tarnow, P. Rossing et al., "Predictors for the development of microalbuminuria and macroalbuminuria in patients with type 1 diabetes: inception cohort study," British Medical Journal, vol. 328, no. 7448, pp. 1105-1108, 2004.

[19] P. Gæde, L. Tarnow, P. Vedel, H.-H. Parving, and O. Pedersen, "Remission to normoalbuminuria during multifactorial treatment preserves kidney function in patients with type 2 diabetes and microalbuminuria," Nephrology Dialysis Transplantation, vol. 19, no. 11, pp. 2784-2788, 2004.

[20] S. Araki, M. Haneda, T. Sugimoto et al., "Factors associated with frequent remission of microalbuminuria in patients with type 2 diabetes," Diabetes, vol. 54, no. 10, pp. 2983-2987, 2005.

[21] J. M. Steinke, A. R. Sinaiko, M. S. Kramer, S. Suissa, B. M. Chavers, and M. Mauer, "The early natural history of nephropathy in type 1 diabetes: III. Predictors of 5-year urinary albumin excretion rate patterns in initially normoalbuminuric patients," Diabetes, vol. 54, no. 7, pp. 2164-2171, 2005.

[22] T. Yamada, M. Komatsu, I. Komiya et al., "Development, progression, and regression of microalbuminuria in Japanese patients with type 2 diabetes under tight glycemic and blood pressure control: the Kashiwa study," Diabetes Care, vol. 28, no. 11, pp. 2733-2738, 2005.

[23] M. E. Molitch, A. I. Adler, A. Flyvbjerg et al., "Diabetic kidney disease: a clinical update from Kidney Disease: improving Global Outcomes," Kidney International, vol. 87, no. 1, pp. 20 30, 2015.

[24] H.-H. Parving, U. Smidt, A. Andersen, and P. Svendsen, "Early aggressive antihypertensive treatment reduces rate of decline in kidney function in diabetic nephropathy," The Lancet, vol. 321, no. 8335, pp. 1175-1179, 1983.

[25] M. Shimizu, K. Furuichi, H. Yokoyama et al., "Kidney lesions in diabetic patients with normoalbuminuric renal insufficiency," 
Clinical and Experimental Nephrology, vol. 18, no. 2, pp. 305312, 2014.

[26] H. Taniwaki, Y. Nishizawa, T. Kawagishi et al., "Decrease in glomerular filtration rate in Japanese patients with type 2 diabetes is linked to atherosclerosis," Diabetes Care, vol. 21, no. 11, pp. 1848-1855, 1998.

[27] R. J. MacIsaac, S. Panagiotopoulos, K. J. McNeil et al., "Is nonalbuminuric renal insufficiency in type 2 diabetes related to an increase in intrarenal vascular disease?" Diabetes Care, vol. 29, no. 7, pp. 1560-1566, 2006.

[28] H. Yokoyama, H. Sone, M. Oishi, K. Kawai, Y. Fukumoto, and M. Kobayashi, "Prevalence of albuminuria and renal insufficiency and associated clinical factors in type 2 diabetes: the Japan Diabetes Clinical Data Management study (JDDM15)," Nephrology Dialysis Transplantation, vol. 24, no. 4, pp.1212-1219, 2009.

[29] G. Penno, A. Solini, E. Bonora et al., "Clinical significance of nonalbuminuric renal impairment in type 2 diabetes," Journal of Hypertension, vol. 29, no. 9, pp. 1802-1809, 2011.

[30] V. Rigalleau, C. Lasseur, C. Raffaitin et al., "Normoalbuminuric renal-insufficient diabetic patients: a lower-risk group," Diabetes Care, vol. 30, no. 8, pp. 2034-2039, 2007. 


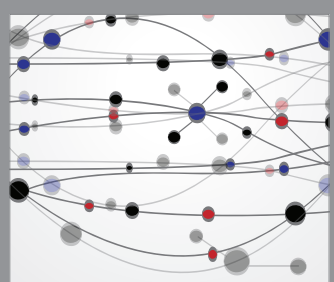

The Scientific World Journal
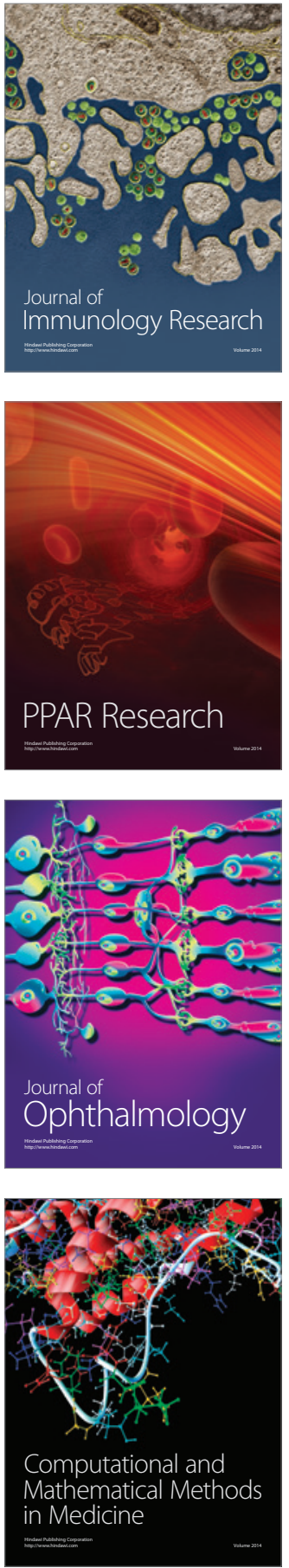

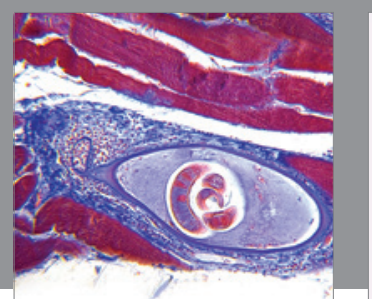

Gastroenterology Research and Practice

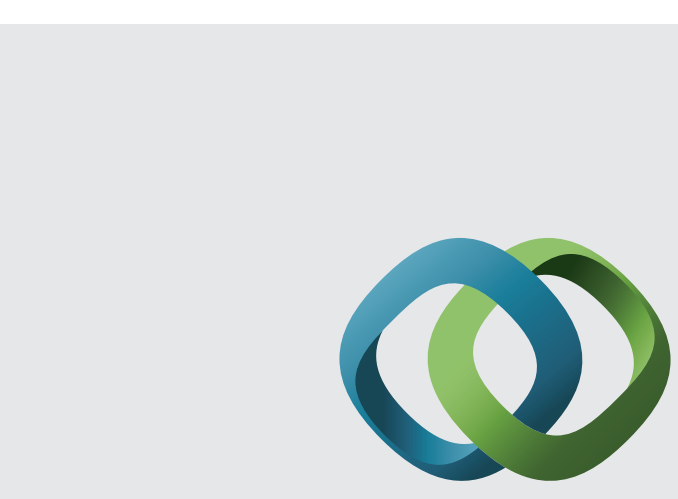

\section{Hindawi}

Submit your manuscripts at

http://www.hindawi.com
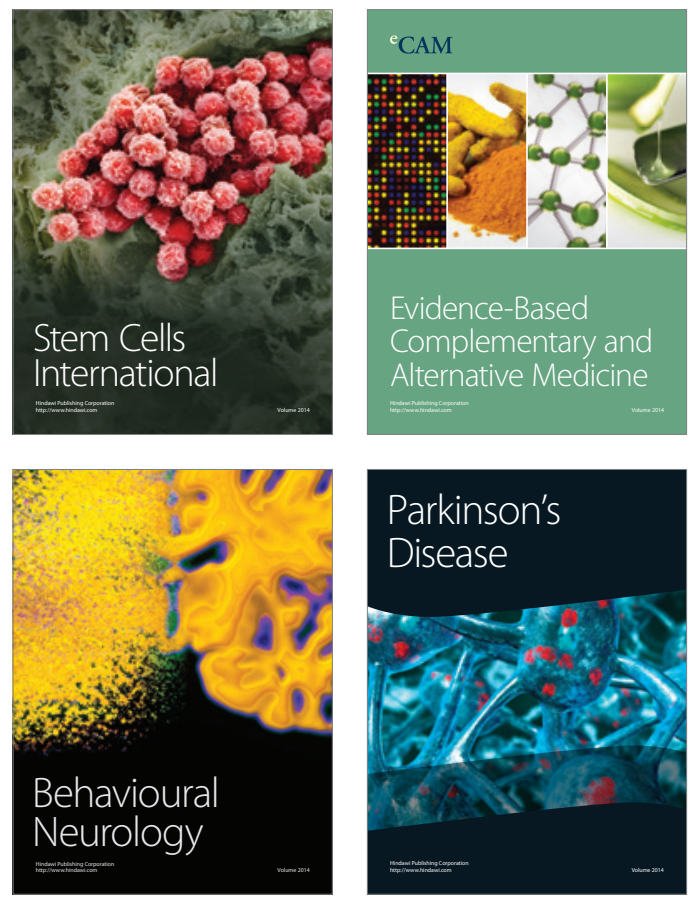
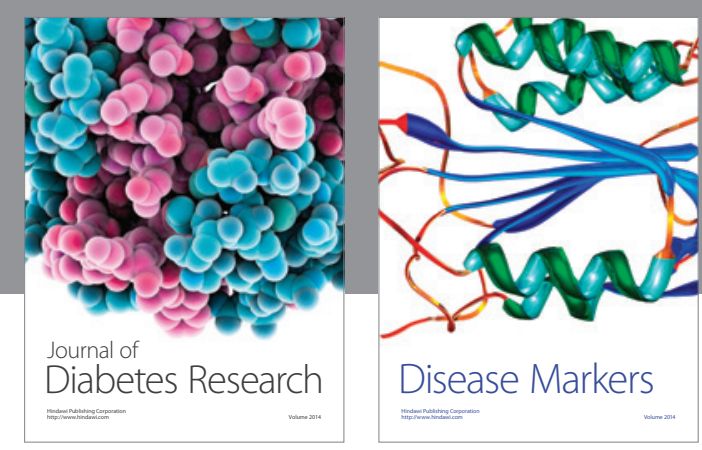

Disease Markers
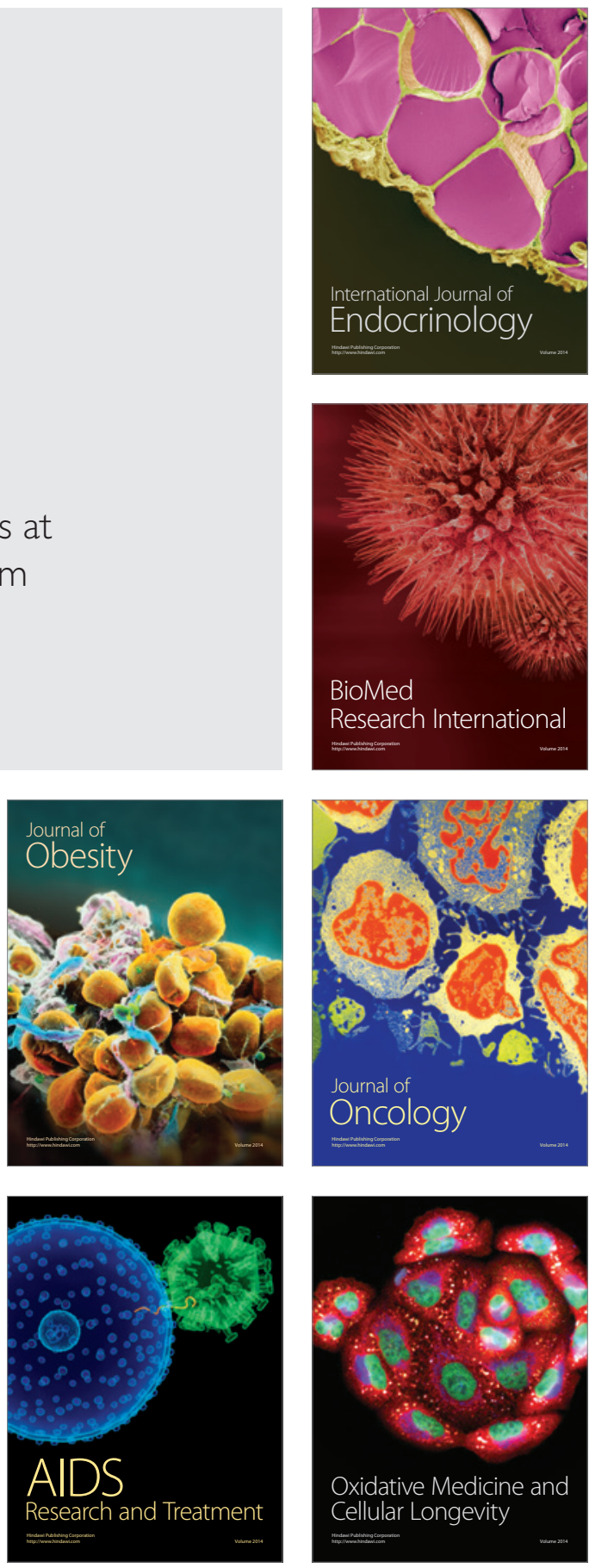